\title{
A NOTE ON SECOND-ORDER NONLINEAR DIFFERENTIAL EQUATIONS
}

\author{
W. R. UTZ ${ }^{1}$
}

In this note we prove a theorem on the behavior, as $t \rightarrow \infty$, of solutions of the nonlinear equation

$$
x^{\prime \prime}+\alpha(x) x^{\prime}+\beta(x) x=0
$$

and prove a corollary to this theorem concerning the behavior of the solutions, as $t \rightarrow \infty$, of

$$
x^{\prime \prime}+g\left(x^{\prime}\right)+c x=0 .
$$

It will be convenient to be able to refer to the following elementary and intuitively obvious lemma in both proofs.

Lemma. Suppose that $x(t)$ is a real function for which $x^{\prime \prime}(t)$ is defined for all $t \geqq a$. (i) If for all $t \geqq a, x^{\prime}(t)<0$ and $x^{\prime \prime}(t) \leqq 0$, then $\lim _{t \rightarrow \infty} x(t)$ $=-\infty$. (ii) If for all $t \geqq a, x^{\prime}(t)>0$ and $x^{\prime \prime}(t) \geqq 0$, then $\lim _{t \rightarrow \infty} x(t)=\infty$.

Throughout the note a function is said to oscillate or be oscillatory when and only when it has arbitrarily large zeros.

THEOREM. If $\alpha(x)$ and $\beta(x)$ are real functions such that for all real $x$,

$$
\alpha(x) \leqq 0, \quad \beta(x)>0
$$

and if $x(t)$ is a solution of (1) valid for all large $t$, then $x(t)$ oscillates or, for all large $t, x(t)$ is monotone. In case $x(t)$ is monotone increasing, $\lim _{t \rightarrow \infty} x(t)>0$ and in case $x(t)$ is monotone decreasing, $\lim _{t \rightarrow \infty} x(t)<0$.

Proof. Suppose that $x(t)$ does not oscillate. Then for large $t, x$ is of fixed sign. We assume that $x>0$ and note that a parallel argument holds for $x<0$. If $x^{\prime}(t)=0$, then $x^{\prime \prime}=-\beta(x) x$ and $x^{\prime \prime}<0$ hence $x^{\prime}(t)$ cannot have arbitrarily large zeros as $x(t)$ would have infinitely many critical values all of which would be maxima. Thus, for $t$ large, $x^{\prime}(t)$ is of fixed sign.

Case 1. If $x^{\prime}<0$ then, since

$$
x^{\prime \prime}=-\alpha(x) x^{\prime}-\beta(x) x,
$$

Presented to the Society, August 24, 1956; received by the editors December 27, 1955 and, in revised form, January 23, 1956.

${ }^{1}$ This research was supported by the United States Air Force through the Office of Scientific Research of the Air Research and Development Command. 
$x^{\prime \prime}<0$ and, by the lemma, $\lim _{t \rightarrow \infty} x(t)=-\infty$ which contradicts $x(t)>0$ for large $t$.

CASE 2. If $x^{\prime}>0$ then $x(t)$ is a montone increasing function and since $x>0, \lim _{t \rightarrow \infty} x(t)>0$.

COROLlaRy. Let $c$ be a positive constant and suppose that $g(0)=0$ and that for all real $z, g^{\prime}(z) \leqq 0$. If $x(t)$ is a solution of (2) valid for all large $t$, then $x(t)$ is oscillatory, $\lim _{t \rightarrow \infty} x(t)=\infty$, or $\lim _{t \rightarrow \infty} x(t)=-\infty$.

Proof. If we set $v=x^{\prime}$, then

$$
v^{\prime \prime}+g^{\prime}(v) v^{\prime}+c v=0
$$

which is (1) with

$$
g^{\prime}(v)=\alpha(v) \leqq 0, \quad c=\beta(v)>0
$$

hence our theorem applies to (3).

If $v \equiv 0$, then $x(t) \equiv 0$ since $g(0)=0$. Hence $x$ is oscillatory.

Suppose that $v$ is oscillatory but $x$ is not oscillatory. Then $v$ has arbitrarily large zeros and $x$ is eventually of fixed sign. When $v=0$, $x^{\prime \prime}=-c x$ and $x$ has extrema for arbitrarily large $t$ which are all maxima or all minima. As this is impossible we conclude that $v$ oscillatory implies $x$ oscillatory.

According to our theorem, if $v$ is not oscillatory, then $v$ is monotone. Assume $v$ is monotone increasing, then, according to the theorem above, eventually $v>0$. Thus for sufficiently large $t, x^{\prime}>0, x^{\prime \prime} \geqq 0$ and by the lemma $\lim _{t \rightarrow \infty} x(t)=\infty$.

Similarly, if $v$ is monotone decreasing, $\lim _{t \rightarrow \infty} x(t)=-\infty$. This completes the proof of the corollary.

Equations (1) and (2) may be considered as generalizations, in two directions, of the linear equation

$$
x^{\prime \prime}+d x^{\prime}+e x=0
$$

with constant coefficients $d \leqq 0, e>0$. Thus we have shown that, for large $t$, solutions of (1) and (2), under the hypotheses of the theorem and corollary, behave as the solutions of (4).

UNIVERSITY OF MISSOURI AND INSTITUTE FOR ADVANCED STUdy 